\title{
Intra-articular operative temporomandibular joint arthroscopy
}

\author{
Howard A. Israel ${ }^{1,2}$ \\ ${ }^{1}$ Touro College of Dental Medicine at New York Medical College, Hawthorne, NY, USA; ${ }^{2}$ Division of Oral \& Maxillofacial Surgery, Cornell \\ University, Weill Cornell Medicine, New York, NY, USA \\ Correspondence to: Howard A. Israel, DDS. Clincal Professor of Dental Medicine, Touro College of Dental Medicine, Adjunct Professor of Clinical \\ Surgery, Weill Cornell Medicine, 19 Skyline Drive, Hawthorne, NY 10532, USA. Email: drhowardisrael@yahoo.com.
}

\begin{abstract}
Numerous intra-articular operative arthroscopic techniques have been reported in the literature since the advent of temporomandibular joint (TMJ) arthroscopy. Ideal randomized controlled trials regarding outcomes are lacking nor is there strong evidence supporting one operative technique over another. However, there has been a vast experience with TMJ arthroscopy over the past several decades with case series, retrospective and prospective outcomes assessments and systematic reviews reported in the literature. Regardless of the intra-articular operative technique there are consistently favorable outcomes in the range of $80-90 \%$. Essential factors to be considered when determining the operative technique include the goals of the surgical procedure based on diagnosis and pathology, and the technical expertise and experience of the surgeon. Operative arthroscopy focused on the removal of adhesions, reduction of synovial inflammation, mobilization of the disc and biopsy/removal of pathological tissue with preservation of relatively healthy tissues are emphasized. In the future the intra-articular arthroscopic procedures to be performed will evolve with advances in technology, education, training, virtual simulation and continued clinical research findings on outcomes. Regardless of future advances, surgeons must be able to acknowledge their individual strengths, weaknesses, level of training, commitment to lifelong learning and need to continually treat each individual patient with the goal of achieving maximum benefit with the least risk.
\end{abstract}

Keywords: Temporomandibular joint (TMJ) arthroscopy; intra-articular; adhesions; synovitis; osteoarthritis

Received: 19 June 2020; Accepted: 16 September 2020; Published: 30 March 2021.

doi: 10.21037/fomm-20-34

View this article at: http://dx.doi.org/10.21037/fomm-20-34

\section{Introduction}

Clinicians treating patients with temporomandibular joint (TMJ) disorders must establish a diagnosis and determine etiologic factors which serve as a basis for treatment. The dilemma of which treatment will work best for patients with a given diagnosis and individual etiologic factors remains controversial. Successful outcomes can be achieved from minimal intervention following the natural course of disease, non-surgical therapies, to the most advanced surgical procedures. The focus here is to assess the best evidence available providing the rationale for operative arthroscopy and identifying essential factors to consider when choosing arthroscopic techniques.

\section{Literature}

When considering an evidence-based approach, prospective randomized controlled studies and systematic reviews with meta-analysis are considered high levels of evidence. Randomized controlled studies are lacking when considering operative arthroscopy for several reasons. With patients who have prolonged, severe symptoms, recruitment for a study with controls is difficult and raises ethical issues when the surgeon has a high success rate with arthroscopy. Performing double blinded studies is impractical, particularly when the surgeon is directly involved with postoperative monitoring/management. Although systematic reviews with meta-analysis regarding 
TMJ surgery have been performed, the literature consists primarily of retrospective or prospective cohort studies (intermediate evidence), chart reviews and case series (lower evidence). Opinion-based case reports and editorials represent the lowest evidence to guide treatment recommendations (1). However, the collective literature enables the development of principles of treatment from the best evidence available regarding surgical options.

The natural course of internal derangement of the TMJ without treatment is often associated with significant resolution of symptoms (2-4) with approximately $25-33 \%$ of patients continuing without improvement (4). Patients with advanced internal derangement and/or osteoarthritis are at higher risk for not improving spontaneously (2-4). Non-surgical therapies are associated with a significant reduction in symptoms the majority of the time. A 10-year follow-up study of patients with disc displacement without reduction, treated with medication, manipulation and appliance therapy revealed $89 \%$ successful outcomes, improved maximum interincisal opening (MIO) and decreased pain (5). Non-surgical therapies for internal derangement have demonstrated improvement in signs and symptoms with no significant differences between treatment and non-treatment groups $(6,7)$. Although most patients with symptomatic internal derangement improve eventually, those with persistent symptoms often develop chronic pain with significantly impaired quality of life.

Since successful outcomes can be achieved with minimal and/or non-surgical therapies, some clinicians have questioned a role for surgical treatment. One study compared surgery to non-surgical therapies and reported no differences in outcomes, although there was no control group without any treatment (8). An excellent review of the role of TMJ surgery by Laskin (9) concluded prospective, randomized, controlled studies are lacking. Evidence for surgery comes mostly from case series with arthrocentesis, arthroscopy, discoplasty, discectomy all reported to have successful outcomes of $80-90 \%$. Surgical success is highest with the first surgery with subsequent surgeries having reduced success rates. Surgical failure is associated with persistence of etiologic factors such as mandibular parafunction (joint overload).

Regardless of the management/treatment studies demonstrate a success rate of $80-90 \%$. In the small percentage of patients with non-surgical therapies and persistent symptoms one cannot predict if or when improvement will occur. Clearly, appropriate surgical intervention must be considered in some patients to prevent complications from progression of symptoms and chronicity.

Systematic meta-analysis has been reported on surgical outcomes for internal derangement to compensate for lack of parallel control groups, comparing arthroscopy, arthrocentesis and discoplasty to historical controls (10). Only arthroscopy and arthrocentesis demonstrated effectiveness significantly greater than all assumed control group improvement rates. Another systematic meta-analysis concluded arthroscopy was more effective in improving joint movement and pain (11). Clinical research, case series and systematic reviews overwhelmingly demonstrate that regardless of the operative procedure, surgical outcomes consistently demonstrated improvement in mandibular range of motion and pain reduction in $80-90 \%$ of cases.

\section{Diagnosis}

A thorough history, examination and imaging is necessary for an accurate diagnosis prior to treatment. The importance of the diagnostic evaluation cannot be over emphasized as surgical failures frequently can be attributed to misdiagnosis. Surgical failures can occur when preoperative MRIs reveal disc displacement but the patient's symptoms are primarily from extra-articular pathologies, such as masticatory myalgia. The gold standard for the diagnostic workup includes a detailed history and clinical examination. In general, patients with true intra-articular pathology have pain that is localized to the involved TMJ, which is increased with mandibular movement or masticatory function. Failure to reproduce pain localized directly to the involved TMJ, stimulated with masticatory load and/or movement, is a warning that the main cause of pain may not be intra-articular, even with MRI confirmed disc displacement. Diagnostic imaging is important, however, imaging results do not necessarily correlate with symptoms and pathology. The surgeon must treat the patient, not the MRI. Studies have revealed MRIs in asymptomatic subjects demonstrate disc displacement in the range of $32-38 \%(12,13)$. MRI studies have shown correlations of pain with joint effusion, an important finding in true intra-articular pathology $(14,15)$. MRIs and CT scans must demonstrate the presence of a joint space for arthroscopy to be performed as joints with ankylosis or neoplasia lacking a joint space require open joint surgery.

The Diagnostic Criteria for Temporomandibular Disorders (formerly Research Diagnostic Criteria) combined with the American Academy of Orofacial Pain 


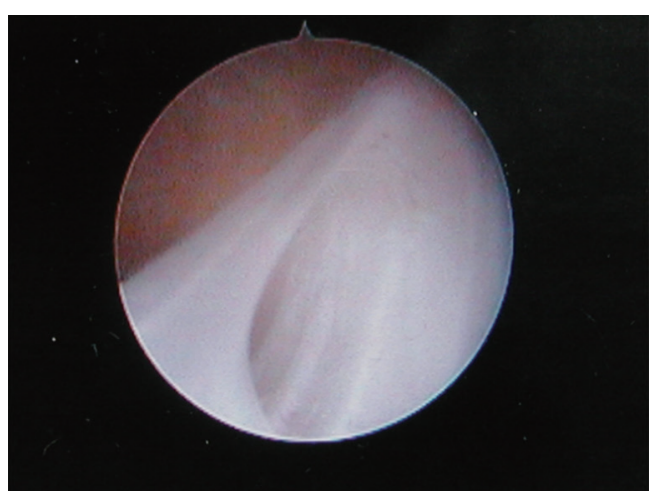

Figure 1 Arthroscopic view of adhesions in the posterior-medial aspect of the superior joint space in a left temporomandibular joint.

Classification has been validated for use in patient care and clinical research. Patients are categorized according to physical signs, AXIS I, and psychosocial status, AXIS II. Most patients who are candidates for surgical treatment have disc disorders, degenerative joint disease, hypermobility/ hypomobility and inflammatory arthropathies (synovitis). Combinations of these physical signs are common as the structure and function of intra-articular tissues become more compromised. Surgeons must determine if intraarticular pathologies are the main cause of symptoms rather than extra-articular conditions such as masticatory muscle disorders. Clinicians must recognize depression and other symptoms due to pain, reduced function, and quality of life. Although the psychosocial status of the patient is extremely important regarding patient management, AXIS II classification does not determine the presence of intraarticular pathology requiring treatment $(16,17)$.

Wilkes Staging classifies joints according to five stages of progression of internal derangement to osteoarthritis. Staging is required to reflect the extent of the disease process. Preoperative staging is particularly important as the surgeon must anticipate intra-articular pathologies and plan the arthroscopy accordingly (18).

Diagnostic arthroscopy provides the most important information determining the intra-articular surgery to be performed. Arthroscopic diagnosis is based on visual inspection of the pathologic intra-articular tissues. The most common intra-articular pathologies are synovitis, adhesions, osteoarthritis, anterior disc position, disc perforation and inflamed synovial plicae. Less common intra-articular pathologies include synovial chondromatosis, pigmented villonodular synovitis, crystalline arthropathies and neoplasia.

\section{Surgical options: major factors}

When considering surgical options, the least invasive surgical approach consistent with the most favorable risk: benefit ratio is indicated as the initial surgical treatment of choice. Arthroscopy, when performed by a trained surgeon is the ideal surgical treatment in cases where pathology does not compromise the joint space. Arthroscopy has significant advantages over arthrocentesis including direct visualization of pathology, lysis of adhesions and tissue removal for a histopathological diagnosis.

Once the surgeon has determined that arthroscopic surgery is to be performed, there are numerous operative techniques available. Important factors determining choice of technique include: (I) preoperative diagnosis and Wilkes staging; (II) diagnostic arthroscopy with specific intraarticular pathologies visualized; (III) major goals of the surgical procedure; and (IV) the surgeon's skill and training.

\section{Intra-articular patbology}

Extensive fibrous adhesions require more advanced arthroscopy to remove pathology (Figure 1). Some patients with pain not clearly muscular or intra-articular in origin undergo arthroscopy. If there is minimal arthroscopic pathology (Figure 2), symptoms are likely muscular and warranting minimal operative maneuvers and fortunately arthrotomy was not performed. Joints with significant degeneration of articular cartilage, synovitis, adhesions, and disc displacement (Figure 3) require operative maneuvers. Some patients with minimal symptoms, have diagnostic images with major pathologic changes, necessitating an arthroscopic biopsy to establish an accurate diagnosis prior to treatment (Figure 4).

\section{Goals of surgery}

The surgeon must determine the specific goals for each patient based on symptoms, clinical diagnosis and pathology visualized arthroscopically. Significant limitation of mandibular range of motion warrants removal of adhesions which frequently create a tether on inflamed synovial tissues. With normal range of motion, severe pain and MRIs demonstrating an effusion, arthroscopic visualization and treatment of the most inflamed synovial tissues is a priority. Patients with loud painful clicking can benefit from arthroscopic disc repositioning surgery. Patients with unusual findings on diagnostic images (MRI and/or CT), 

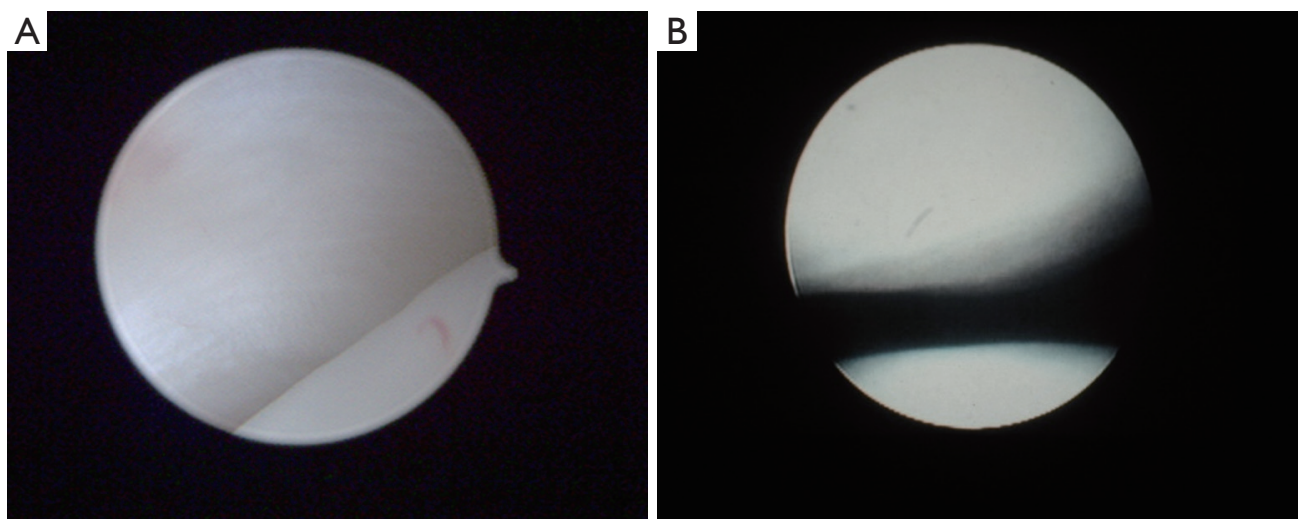

Figure 2 Normal intra-articular tissues. (A) Right temporomandibular joint glenoid fossa and retrodiscal tissues without evidence of synovitis. (B) Right temporomandibular joint articular eminence and disc with normal cartilage and normal disc position.
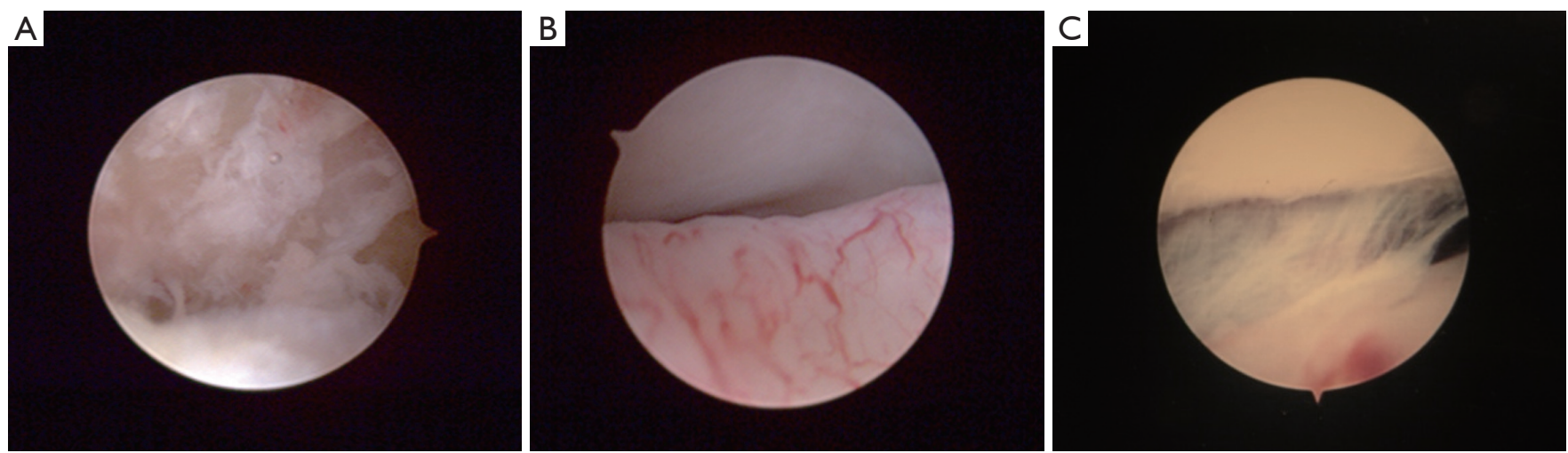

Figure 3 Pathologic intra-articular tissues. (A) Advanced osteoarthritis with full thickness degeneration of cartilage articular eminence left temporomandibular joint superior joint space. (B) Anterior disc position and vascular retrodiscal tissues with synovitis roofing the condyle left temporomandibular joint superior joint space. (C) Adhesions anterior left temporomandibular joint superior joint space.
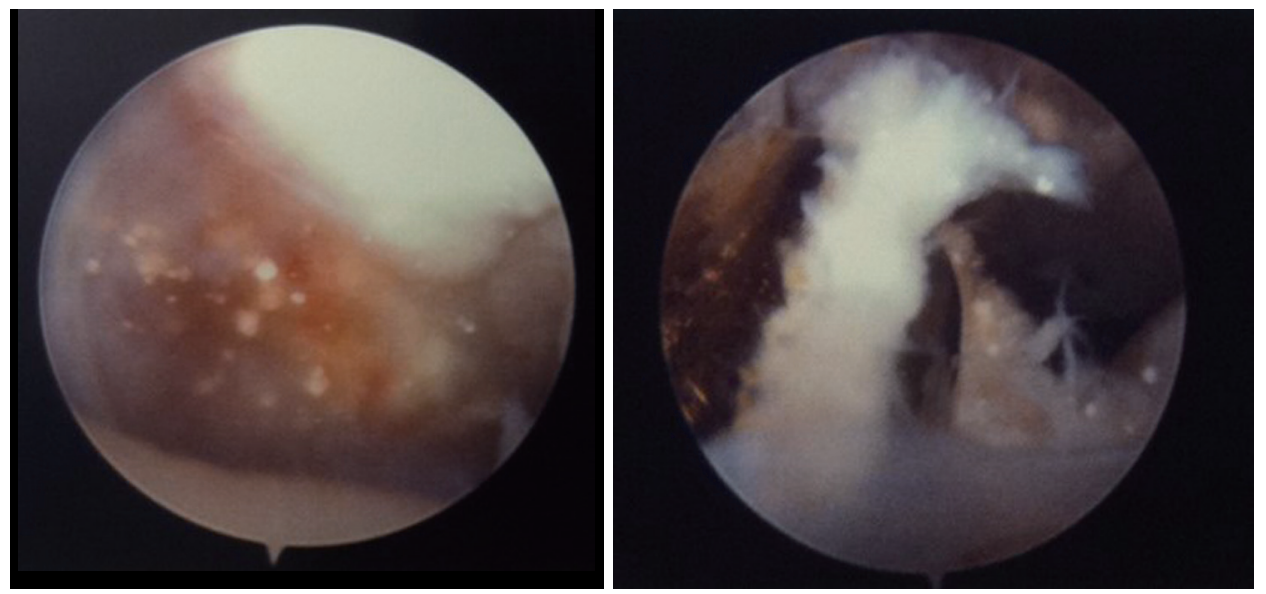

Figure 4 Crystal deposition in synovium posterior synovial pouch right temporomandibular joint. Arthroscopic biopsy confirmed calcium pyrophosphate dihydrate crystals and a diagnosis of chondrocalcinosis. 
bone resorption and/or atypical pathologic tissues seen arthroscopically require biopsy to establish a histopathologic diagnosis. Many patients have combinations of pathologies and symptoms, requiring the surgeon to perform multiple operative techniques to address each problem.

\section{Training and skill of the oral \& maxillofacial surgeon}

Due to the nature of arthroscopy, the skills required for technical proficiency are vastly different from other surgical procedures. Surgery requires direct visualization anatomy at the surgical site and proprioceptive feedback to the surgeon's hands. With arthroscopy, the proprioceptive feedback enables the surgeon to envision the correct anatomical landmarks without direct visualization to permit joint entry. Operative arthroscopy involves minute movements of the instruments magnified as major movements on the monitor. Arthroscopy requires the surgeon to be able to place small surgical instruments within the visual field of the arthroscope, with successful triangulation permitting operative maneuvers to be performed. Teaching these skills, as well as the ability to envision where two objects will meet in a small space is difficult and requires repetition. Many oral and maxillofacial surgery training programs are limited in providing this training.

There are fellowships and high-quality courses that provide the necessary didactic knowledge and clinical skills to train surgeons to become proficient with TMJ arthroscopy at various levels. A fellowship in which there is repeated clinical training with experienced arthroscopic surgeons is ideal. Courses conducted by experienced arthroscopic surgeons with regularly concentrated sessions for several days throughout the year can successfully train surgeons in basic TMJ arthroscopy (single puncture) progressing gradually to more advanced techniques (double puncture with triangulation). Successful training requires didactics and appropriate sequencing of clinical skills, from hands on experience with anatomical models, cadaver sessions, assisting in surgery, and ultimately direct patient care supervised by the experienced arthroscopist. Undoubtedly, virtual simulation will increasingly become an educational technology which will further our progress in training oral and maxillofacial surgeons in the future. Although technical skills are essential when learning operative arthroscopy, equal emphasis must be placed on diagnosis and identification and control of the etiologic factors (e.g., joint overload from parafunction, systemic diseases) which cause the disease process.

\section{Intra-articular operative arthroscopic techniques}

The basic operative techniques that have been described are as follows:

\section{Single puncture}

* Arthroscopic lavage (AL) — diagnostic arthroscopy with lavage (Level I)

* Arthroscopic lysis and lavage (L\&L) - diagnostic arthroscopy, lavage \& "blind sweep" (Level I)

* Arthroscopic lysis, lavage, needle working instrument (Level II)

\section{Double puncture with triangulation}

* Treatment/removal of pathology/debridement (Level III)

* Disc repositioning (Level III)

Surgeons have described three levels of TMJ arthroscopy, Level I the most basic, involving a single puncture with diagnostic evaluation and lavage. Level I L\&L includes a "blind sweep" of the superior joint space using a blunt instrument aimed at lysis of adhesions. Level II involves a single puncture, with the irrigation needle used for minor operative maneuvers including visualized lysis of adhesions or targeted-tissue medication injections. Level III includes more advanced procedures, double punctures, triangulation and visualized intraoperative maneuvers, including removal of pathology, debridement, motorized shaving, synovial biopsies, disc mobilization or disc repositioning with stabilization (discopexy). Some advanced procedures require a triple puncture. Arthroscopic eminoplasty, treatment of condylar fractures, and other infrequently performed advanced techniques are beyond the scope of this review.

\section{$A L$, artbrocentesis and lysis \& lavage}

Surgeons at an early stage of development of operative skills begin treating patients with diagnostic AL. The surgeon must have skills to routinely place a trocar with cannula into the superior joint space without creating damage to adjacent structures (ear, brain, cartilage, and facial nerve). An outflow portal must be placed to permit irrigating fluid (Normal Saline, Lactated Ringers Solution) to flow into and out of the joint space. Continuous irrigation is required to permit clear images on the monitor, otherwise bleeding will obstruct or greatly decrease image clarity. Aside from 


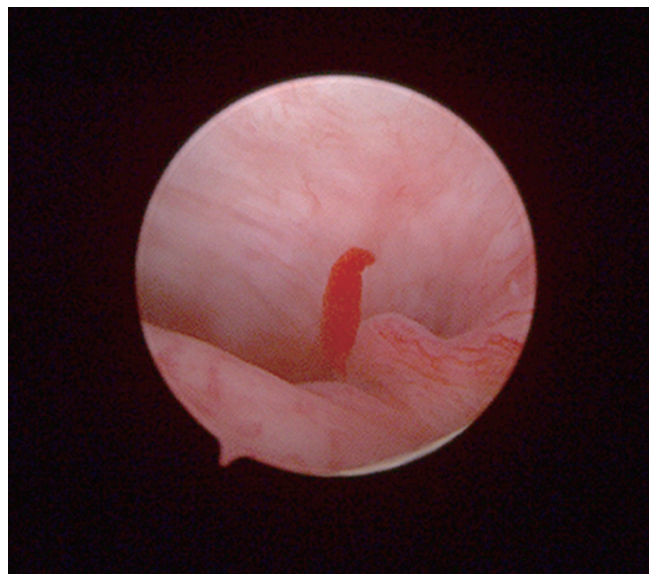

Figure 5 Hypertrophic synovitis retrodiscal tissues and posterior synovial pouch left temporomandibular joint superior joint space.

providing the ability to visualize tissues, from a therapeutic standpoint, AL is essentially the same as arthrocentesis except the arthroscopic cannula is larger than the inflow needle with arthrocentesis. With AL and lysis \& lavage there is no second portal/cannula for instrument entry permitting visualized intra-articular surgery.

The literature on outcomes of AL generally demonstrate improvement in $\mathrm{MIO}$ and reduction in pain. Investigators performed ultrathin arthroscopy with lavage on closed lock patients, with significant improvement in MIO and pain reduction and success rates of $80 \%$ (19). Other AL studies demonstrated successful outcomes of $67 \%(20)$ and $60 \%$ (21). A systematic review of randomized controlled trials comparing lavage to non-surgical therapy demonstrated better pain relief with lavage, but no difference in improvement of MIO (22). One systematic review of arthrocentesis, although acknowledging research methodology deficiencies, reported a success rate of $83.2 \%$ in all combined studies including 571 joints with closed lock (23). Another review of arthrocentesis (24) reported an $80 \%$ successful outcomes rate. Therefore, the best evidence in the literature on arthrocentesis or $\mathrm{AL}$, including systematic reviews and case series, reflects successful outcomes in $60-80 \%$ of cases.

$\mathrm{L} \& \mathrm{~L}$ involves "blind sweep" of the superior joint space with a blunt probe following diagnostic arthroscopy. The most common TMJ arthroscopic technique performed is $\mathrm{L} \& \mathrm{~L}$ with a significant literature on outcomes. Studies report consistently successful outcomes with $L \& L$ in the range of $80-90 \%$ with significant improvement in $\mathrm{MIO}$ and pain (25-34). Variations of the L\&L technique, include lateral eminencia release and capsular stretch $(26,27)$. One study (35) of arthroscopic anterolateral capsular release with Holmium: YAG laser or electrocautery on 152 TMJs with internal derangement and osteoarthritis reported successful outcomes from 93-96\%. Moses has emphasized the lateral eminencia release with a capsular stretch maneuver to facilitate joint mobility impaired by adhesions and impingement of the synovium (26). Successful outcomes with significant improvements in pain and MIO occurred in $92 \%$ of patients. Despite improvement, preoperative and postoperative MRIs did not demonstrate any change in disc position. An endaural approach has been reported to facilitate lateral eminencia release and capsular stretch techniques (36). These studies emphasize treatment of fibrous adhesions, regardless of the instrument used, was an important goal of arthroscopy.

A pilot study of 40 patients randomly assigned to one group with routine L\&L and a second group adding Sodium Hyaluronate (HA) lavage demonstrated significant pain reduction in Wilkes Stages III and IV in the HA group (37) warranting further investigation. As surgeons become more experienced with arthroscopy, innovative techniques have emerged, although there is no conclusive evidence that variations of $L \& L$ yield better surgical outcomes.

From an historical perspective, L\&L provided clinical researchers direct viewing of pathologic intra-articular tissues and the opportunity for synovial fluid research (1980s-1990s), increasing knowledge of pathogenesis of inflammatory/degenerative TMJ disorders. Aside from disc displacement, surgeons commonly visualized synovitis and adhesions. Inflamed synovium contains connective tissues with sensory innervation and a significant vascular network (Figure 5). The combination of persistent inflammation and reduced mobility ultimately results in the formation of adhesions, further reducing mobility. Motion is necessary for synovial fluid to provide chondrocyte nutrition, thus reduced mobility causes articular cartilage degradation. Failure of cartilaginous and synovial tissues ultimately leads to altered biomechanics and disc displacement (16,38-44). Synovial fluid research has revealed many biomarkers for inflammation and cartilage degradation, demonstrating that biochemical and tissue changes cause biomechanical instability. Increased understanding of the structure and function of intra-articular tissues has advanced arthroscopic surgery with major goals being removal of adhesions, disc mobilization and reduction of inflammation. 


\section{Disc repositioning}

There are numerous publications describing operative techniques for repositioning an anteriorly displaced disc through an arthroscopic approach (45-50). The arthroscopic disc suturing technique, also referred to as discopexy, requires advanced arthroscopic skills. Although there are variations in the description of arthroscopic discopexy, the basic components of this procedure include a double puncture, triangulation, lysis of adhesions, mobilization of the disc, an anterior releasing incision at the junction of the pterygoid muscle shadow and the anterior synovial recess, and placement of a disc suture or anchor designed to stabilize the disc with the posterior band directly over the head of the condyle. Successful outcomes of arthroscopic disc repositioning have been reported in the range of 69$92.4 \%$ with respect to reduction in symptoms. One study demonstrated an overall success rate of $69 \%$ including all Wilkes stages. Successful outcomes occurred in $87 \%$ of stage II and III patients and $25 \%$ of stage IV and V patients (47). Only two studies demonstrated a postoperative improvement in disc position following discopexy. One study of 764 joints with MRIs 1-7 days postoperatively demonstrated good disc position in $92.4 \%$ (48). Another study demonstrated successful improvement in disc position in 13/16 joints on MRIs taken one year postoperatively (50).

\section{Treatment/removal of pathology}

Although "treatment/removal of pathology" has not been classified as a specific operative arthroscopic technique, a comprehensive review of the literature reveals that virtually all double puncture operative procedures have treatment/removal of pathology as a major component. The conceptual basis of this category of operative arthroscopy is based primarily on the specific goals of the surgery and performing intra-articular procedures designed to achieve these goals. The main concepts that dictate the operative maneuvers with treatment/removal of pathology are:

(I) All intra-articular tissues have structure/function designed to permit functional loading and motion.

(II) Visualization of abnormal intra-articular tissues dictate the operative maneuvers required to restore/ facilitate function.

(III) Damage/removal of functional intra-articular tissues must be avoided.

Essential steps of operative arthroscopy focused on treatment/removal of pathology are described in the following sections.

\section{Diagnostic arthroscopy}

Diagnostic arthroscopy is performed initially, with a single puncture technique and an outflow portal with a \#18-gauge needle. The surgeon carefully inspects the superior joint space for evidence of pathology and/or damaged intraarticular tissues. This is an important first step as it provides the basis for the surgical maneuvers that will be take place during the operative phase of the procedure. This initial diagnostic arthroscopy also allows the surgeon to determine if the preoperative MRIs and clinical examination findings are consistent with the observed intra-articular pathology. In cases where diagnostic arthroscopy reveals minimal tissue damage, the surgeon may consider that the initial clinical diagnosis of intra-articular pathology may not be accurate, and in fact, symptoms may be caused by other conditions such as masticatory muscle disorders. In these situations, the initial diagnostic arthroscopy may be the only procedure performed, without any second portal or intra-operative manipulation. The overriding factor in determining the surgical maneuvers required in cases where there is minimal intra-articular pathology is to preserve these intraarticular tissues without damage or unnecessary operative manipulation.

\section{Progression from diagnostic arthroscopy to Level II}

A variation of this technique can be performed when there is mild degree of intra-articular pathology, such as a low-grade synovitis, or a few loose adhesions. In these circumstances the surgeon has the option of using the \#18-gauge outflow needle as an operative instrument (Level II) without progression to more advance procedures. The surgeon must use a triangulation technique and therefore it is helpful to connect the barrel of a 3-cc syringe to the needle to facilitate positioning of the needle within the visual field of the arthroscope. A limited number of loose adhesions can be lysed with the sharp edge of the needle. Areas where there is a low-grade synovitis can be localized and using a long \#25-gauge spinal needle a steroid can be injected under direct vision into inflamed synovial tissues (Figure 6).

\section{Adhesions}

Adhesions are bands of fibrous connective tissue that restrict translation in the superior joint space. Studies involving intra-articular biochemical changes and pathophysiology $(51,52)$ have demonstrated that inflammatory mediators and 
free radicals are associated with adhesion formation. Joint overload, inflammation of tissues, and lack of joint mobility are external factors that contribute to the biochemical changes and tissue damage. One arthroscopic study revealed mandibular parafunction patients had significant osteoarthritis (cartilage degradation) associated with adhesions (53). Adhesions restrict joint mobility and are frequently found in areas of significant inflammation often appearing as a wall of adhesions involving the posterior recess (Figure 7). An isolated adhesion is sometimes seen invaginating into the superior joint space, and if lined by synovium, represents a synovial plica (Figure 8). These adhesions not only restrict mobility but cause pain when there is impingement on these structures. The removal of

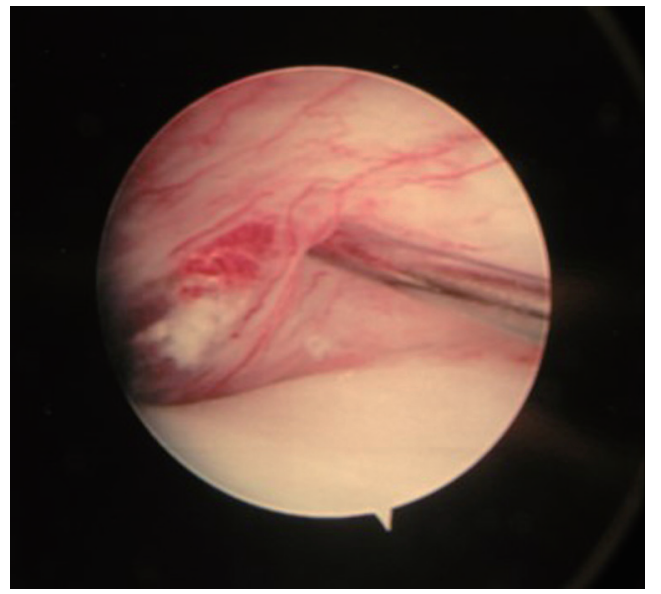

Figure 6 Direct injection into synovitis with \#25 gauge spinal needle with deposition of steroid (Dexamethasone $2 \mathrm{mg}$ ). adhesions under direct vision is important for improvement in mandibular range of motion. A double puncture technique with triangulation permits surgical release of these adhesions with either a blunt or hooked probe for loose thin adhesions. For thick adhesions, a blade, scissor, or grasper are required to remove the adhesion. Other more advanced techniques using the Holmium: Yag laser (35) or coblation (54) have the advantage of reducing intra-articular bleeding. Regardless of the technique used, it is very important for the surgeon to realize that postoperative rehabilitation which emphasizes mobilization exercises are necessary to prevent adhesions from reoccurring to maintain joint mobility (55). Visualization of lysis and removal of adhesions represents a significant advantage of operative arthroscopy over arthrocentesis and AL.

\section{Obtaining a tissue specimen}

Obtaining a tissue specimen is an important goal of operative arthroscopy. It is important to confirm and correlate the appearance of pathologic tissue changes seen arthroscopically with a histopathologic diagnosis. A recent study by Hakim et al. (56) has emphasized the need to correlate arthroscopic biopsy results with the pathologic tissue morphology that is seen, as this will often provide important clues to the etiology of the disease process. This retrospective study of arthroscopic biopsies of synovium in 210 patients demonstrated that a lymphoplasmacytic infiltrate correlated with petechiae synovitis, strongly suggesting that these patients had an immune mediated synovitis and most likely a systemic etiology. Further prospective studies correlating histopathologic and
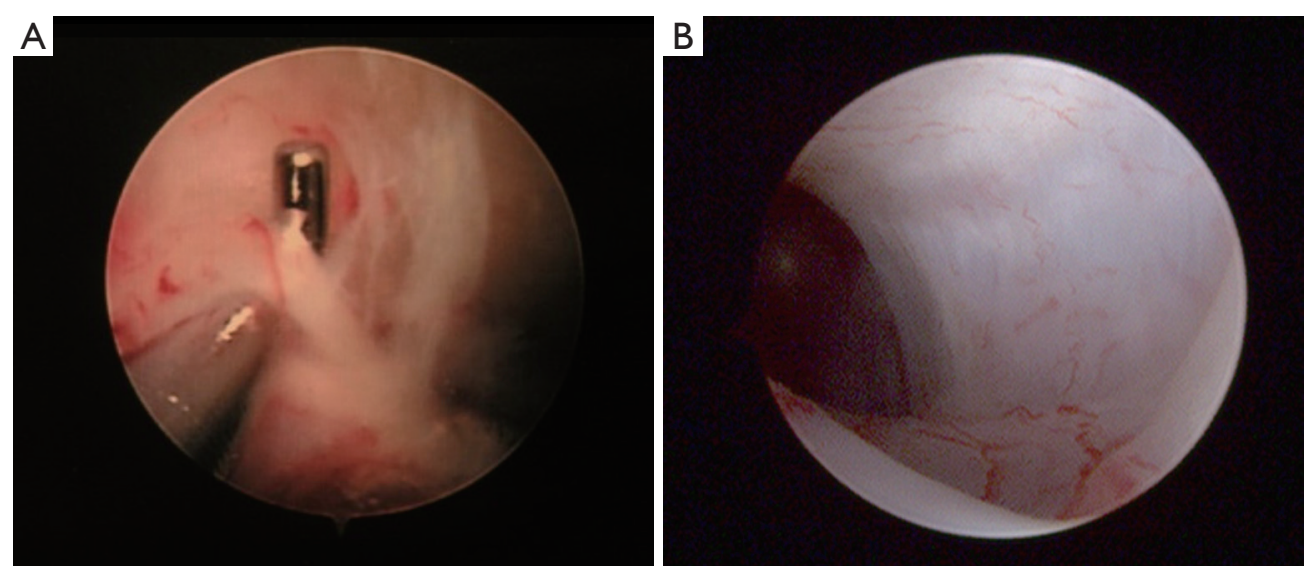

Figure 7 Adhesions. (A) Adhesions forming in areas of synovitis right temporomandibular joint posterior recess; (B) wall of adhesions forming in areas of synovitis left temporomandibular joint posterior medial recess. 


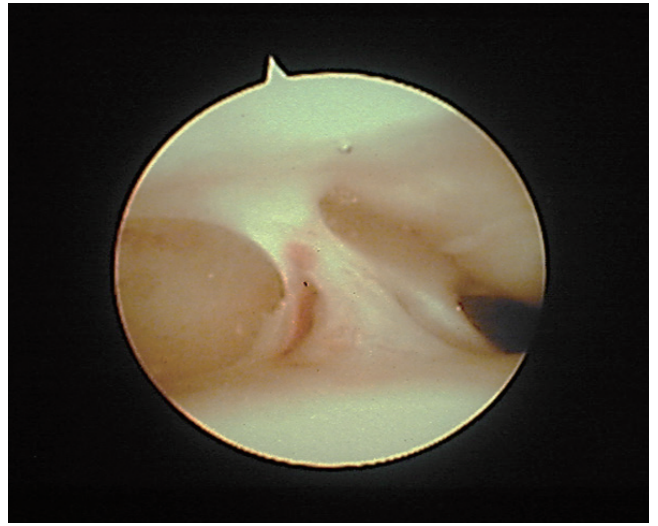

Figure 8 Synovial fold medial wall of left temporomandibular joint with inflammation and fibrosis.

arthroscopic findings can greatly enhance our knowledge of etiologic factors associated with TMJ pathology.

The importance of arthroscopic biopsies cannot be overstated. The TMJ is susceptible to the same pathologies as other synovial joints and patient signs/symptoms are not diagnosis specific. MRI findings that are atypical represent a specific clinical situation where arthroscopic biopsy is essential to establish a diagnosis. These patients may not have significant symptoms; however, the clinical and MRI findings are suggestive of a pathologic process that is atypical. Arthroscopic biopsies can confirm the diagnosis of a neoplastic process (e.g., synovioma, metastatic lesion), a systemic process (e.g., crystalline arthropathy), and other pathologies such as synovial chondromatosis and pigmented villonodular synovitis. The ability to biopsy tissue represents a significant advantage of operative arthroscopy over arthrocentesis.

\section{Osteoarthritis}

Osteoarthritis is visualized in joints that undergo cartilage degradation. Joint overload is a contributory factor in the degradation of cartilage as evidenced by the increased levels of glycosaminoglycans in synovial fluids from joints that demonstrated arthroscopic osteoarthritis $(38,40,43)$. Arthroscopic osteoarthritis occurs more frequently with advancing age (57) due to the amount of time that these joints have been subjected to overload. These patients are prone to joints with Wilkes stages IV and V and disc perforation is common. Studies have reported motorized shaving and/or bipolar cautery of fibrillation tissue for arthroscopic treatment of advanced osteoarthritis $(58,59)$. They demonstrated significant improvements in pain and
MIO in patients with disc perforation.

Arthroscopic treatment of joints with osteoarthritic changes involves direct visualization of the fibrillation tissue of the articular cartilage, disc, or disc perforation. Removal and/or debridement with a motorized shaver or other modality (e.g., Holmium: Yag laser, coblation) is an advanced technique. Care must be taken not to damage the full thickness of articular cartilage and only remove the pathologic cartilage seen as fibrillation that is protruding into the joint space. Importantly, there are no controlled studies that have demonstrated better surgical outcomes with or without removal of the osteoarthritic fibrillation tissue.

\section{Mobilization of the disc}

Mobilization of the disc is an important goal of arthroscopy and is required for normal translation in the superior joint space. Although some have considered reduction of an anteriorly displaced disc and securing that disc position as an important surgical goal, there currently is no clear evidence that better outcomes are achieved. Numerous studies have demonstrated excellent outcomes without a change in disc position. The presence of anteriorly displaced discs on MRIs of asymptomatic subjects $(12,13)$ provides further evidence that disc reduction is not necessary. Studies are lacking that consistently demonstrate a surgically reduced disc will be maintained in the reduced position. The necessity of surgical disc reduction continues to remain controversial.

A frequent arthroscopic observation, particularly in younger patients with anterior disc displacement, is the presence of remodeled retrodiscal tissue over the roof of the condyle. The presence of small vascular channels within this cartilage-like tissue suggests that this tissue originated from synovium and represents remodeled retrodiscal tissues (Figure 9). Retrodiscal tissue consists of synovium overlying rich vascular connective tissue and has the capacity to produce glycosaminoglycans and proteoglycans when stimulated by functional loads (60-62). This gives remodeled retrodiscal tissues the appearance and function of cartilage.

Achieving mobility of the disc is an important goal and is performed following arthroscopic procedures such as lysis/removal of adhesions, debridement of osteoarthritic fibrillation tissue and biopsy of pathologic tissue. Using a double puncture triangulation technique, a blunt probe is advanced medially to the oblique protuberance, a fold of retrodiscal synovial tissue located medially, posteriorly and visualized when the condyle is anteriorly positioned 

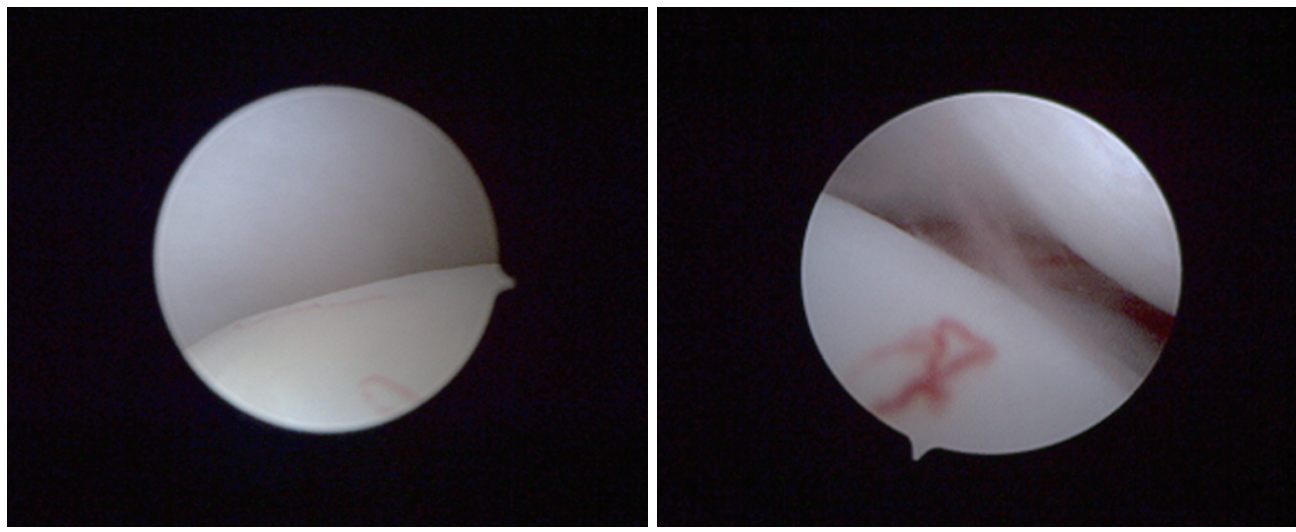

Figure 9 Remodeled retrodiscal tissue roofing condyle. The tissue resembles cartilage but the presence of blood vessels confirms the origin is synovium.
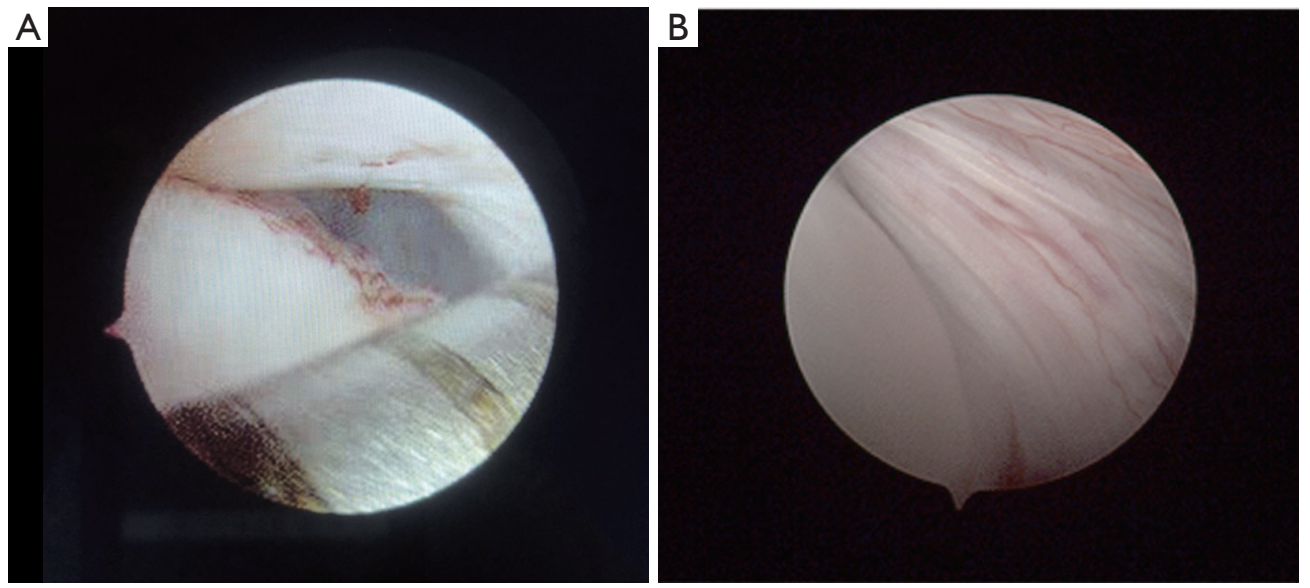

Figure 10 Disc mobilization. (A) Inferior traction to retrodiscal tissues and mobilization of the disc; (B) Inferior traction maintained to mobilize disc with the posterior band now over the roof of the condyle. This position of the disc will not be maintained once inferior traction is released but mobilization has been achieved.

(Figure 10A). Inferior traction on this tissue will mobilize the disc and by maintaining this position with the blunt probe, one can see the posterior band of the disc positioned over the roof of the condyle (Figure 10B). Although this position is not maintained once the inferior traction of the blunt probe is released, disc mobilization has been achieved.

\section{Synovitis}

Synovitis is a common finding in patients with true intraarticular pain. The rich network of sensory nerves in the connective tissue of the synovium accounts for the pain that the patient experiences when there is significant inflammation within the synovial tissues. Synovitis may range from mild to severe depending on the number of dilated blood vessels and the extent of erythema and swelling of the synovial tissues. The inflamed synovial tissues have a variable appearance and may extend over the surfaces of cartilage (Figure 11) or may have a punctate appearance (Figure 12). A significant synovitis within the small joint space of the TMJ can result in a cycle of persistent inflammation and tissue damage, as the patient attempts to apply normal masticatory loads on inflamed synovial tissues. Pathologic changes in the synovium represent a key component in the pathogenesis of many joint diseases. The release of inflammatory mediators has been demonstrated in synovial fluids, with persistent loading of these tissues creating further tissue damage and inflammation. An important component of operative 


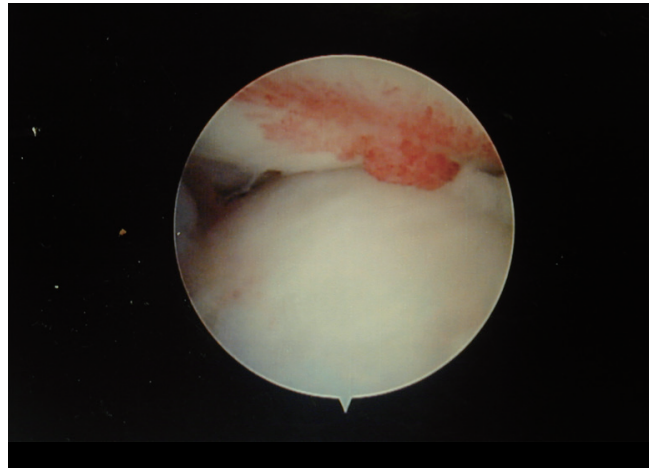

Figure 11 Synovitis creeping over the cartilage of the lateral aspect of the articular eminence of a right temporomandibular joint.

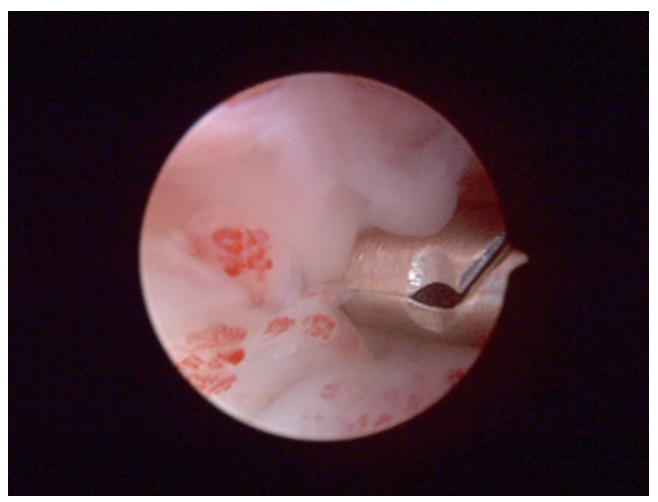

Figure 12 Biopsy of punctate synovitis.

arthroscopy is direct injection of a steroid into the most inflamed synovial tissues. This requires a second portal, with triangulation that permits visualization of the inflamed synovial tissues, and a direct injection using a \#25-gauge spinal needle. Dexamethasone $(4 \mathrm{mg} / \mathrm{mL})$, Betamethasone (6 $\mathrm{mg} / \mathrm{mL}$ ), or Methylprednisolone $(40 \mathrm{mg} / \mathrm{mL}$ ) can be administered with equal effectiveness. A mild ballooning of the synovial tissues following the injection of $0.5-1 \mathrm{~mL}$ of steroid is often visualized. Multiple areas of inflammation require multiple injections. The most common locations for synovial injections are the retrodiscal tissues, the anterior synovial recess, and the lateral synovial recess. The technique described here is not the same as a lavage of the joint space with a steroid as the goal is to place the medication directly into the inflamed synovial tissues. Generally, synovial injections are performed at the end of the arthroscopic procedure.

\section{Mandibular hypermobility with chronic dislocation}

Mandibular hypermobility with chronic dislocation that fails to self-reduce can be treated with arthroscopic TMJ surgery. The basic concept of arthroscopic treatment is to create scarification of retrodiscal tissues to reduce translation of the condyle beyond the articular eminence. A prospective study of 39 dislocation patients who underwent sclerosing injections into the oblique protuberance reported significant improvements in pain, function, and joint loading (63). One study reported cauterization of the retrodiscal tissues for dislocation in 16 patients with a success rate of $95 \%$ after 86 months (64). Variations of the technique have been reported but there has been no consensus on the best technique. For severe cases that do not respond to arthroscopic procedures, eminoplasty is an option considered by surgeons.

\section{Postoperative rehabilitation management}

The importance of appropriate postoperative rehabilitation following surgery cannot be overemphasized. Patient education/guidance directed by the surgeon can determine success or failure. Regardless of operative technique, surgeons must ensure a rehabilitation regimen with control of etiologic factors that result in biochemical and biomechanical instability. There is nothing inherent in the performance of arthroscopy that changes the etiologic factors causing the pathology. Preoperatively the patient must be aware that arthroscopy is not a "quick fix" with a return to normal function within days or weeks. Arthroscopy on all joints require rehabilitation, generally lasting for months focused on: (I) minimizing joint loading, (II) passive motion exercises, (III) control of etiologic factors, (IV) reduction of pain and inflammation.

Excessive joint loading, from parafunctional habits is a common etiologic factor contributing to cartilage degradation exceeding repair. Patients are placed on a nonchew diet for several weeks, with a very gradual return to a diet restoring masticatory function. Absence of pain during mastication is a key factor determining the progression to a more normal diet. If there is pain during mastication the patient returns to a diet that does not cause pain. Aching of masticatory muscles following mastication is common, expected, and due to muscle disuse atrophy. As the patient gradually returns to a more normal diet with function, masticatory muscle strength will gradually return $(39,55)$.

Passive motion exercises to restore range of motion are 
crucial and are performed up to 4 times daily following the procedure. Mobilization of synovial joints is necessary for the pumping action of synovial fluid to provide nutrition to the chondrocytes in articular cartilage, without which cartilage degradation will occur. Exercises are also necessary to prevent reformation of adhesions. Passive motion involves joint movement, without activating surrounding muscles. Finger jaw stretching exercises, the use of passive motion devices and other techniques are required with exercise periods lasting 10 minutes up to four times daily. Physical therapy (PT) is helpful but it is important for the surgeon to emphasize that PT sessions are not to replace passive motion exercises performed regularly by the patient. Exercises and PT that increases joint load must be avoided. Generally, muscle strength will be regained as the patient gradually returns to normal masticatory function. Surgeons are quite familiar with medications designed to reduce pain and inflammation and so this does not require any significant discussion in this review $(39,55)$.

\section{Discussion}

The question remains what is the most appropriate arthroscopic procedure based on a given diagnosis? The literature consistently confirms $80-90 \%$ successful outcomes regardless of operative arthroscopic technique. Major factors which influence this decision are based on answers to several important questions.

\section{What are the goals of the surgery based on the patient's individual clinical diagnosis and artbroscopic patbologic findings?}

For patients with very atypical pathologic MRI findings, a major goal would be arthroscopic biopsy to establish a histopathologic diagnosis, leading to the appropriate treatment. In patients who present with excellent range of motion and loud, painful clicking, the surgeon may consider advanced operative arthroscopy that focuses on the disc, with mobilization or reduction. For mandibular hypomobility patients lysis/removal of adhesions is extremely important. Patients with intra-articular swelling and MRI findings demonstrating a synovial effusion, will require treatment of synovitis as a major priority. Most patients have combinations of these findings and the surgeon must adapt the technique based on direct visualization of pathologic intra-articular tissues.

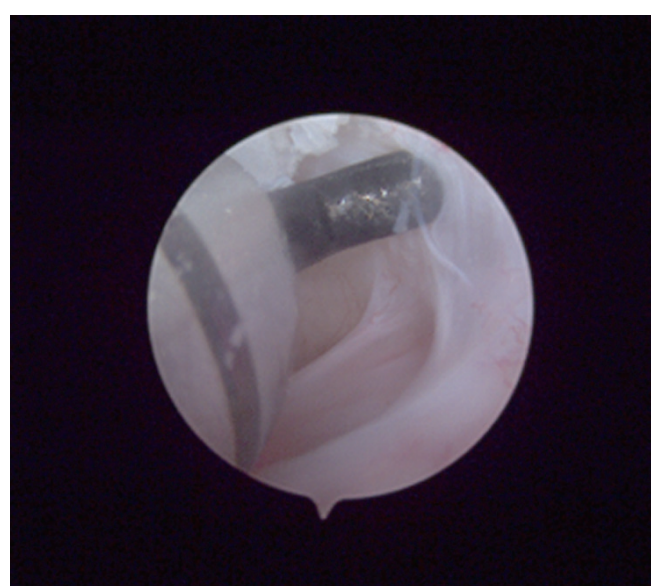

Figure 13 Blunt probe used to lyse loose adhesions posterior recess left temporomandibular joint superior joint space. This is an important step in the progression of training and skill of the oral and maxillofacial surgeon.

\section{What level of arthroscopic skills and experience does the surgeon bave?}

A major consideration when performing arthroscopy is to maintain the structure and integrity of the intra-articular tissues, avoiding damage to these tissues and surrounding anatomic structures. During the early phases of arthroscopic training, the surgeon must master joint entry and diagnostic arthroscopy with lavage. As the surgeon becomes more skilled, operative arthroscopy requires consistency with a double puncture technique and triangulation, permitting visualization of instruments within the field of vision of the arthroscope. As surgeons advance in their training basic operative maneuvers must be mastered such as direct lysis/ removal of adhesions and biopsy (Figure 13). The ability to perform a direct injection of steroid into inflamed synovial tissues is an important operative maneuver in the treatment of synovitis. More advanced procedures require a surgeon with experience and skills that continue to be maintained. Procedures such as discopexy, motorized shaving of osteoarthritic fibrillation tissue, debridement around a perforated disc, biopsies, the removal of pathologies such as synovial chondromatosis require advanced technical skills and surgical judgement. Regardless of the level of training and experience, each surgeon must be acutely aware of their limitations to avoid iatrogenic injury to intra-articular tissues that appear to be relatively healthy.

Arthroscopic treatment of patients with symptomatic 


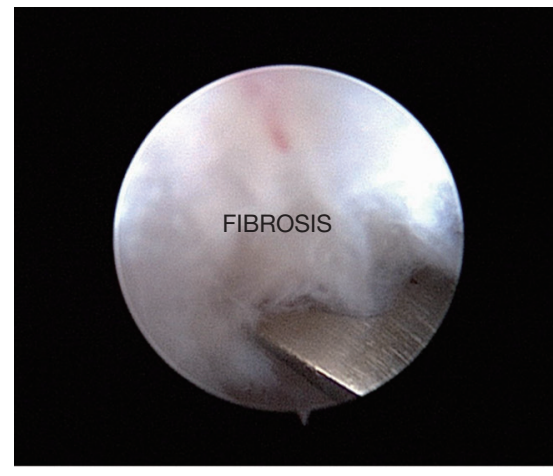

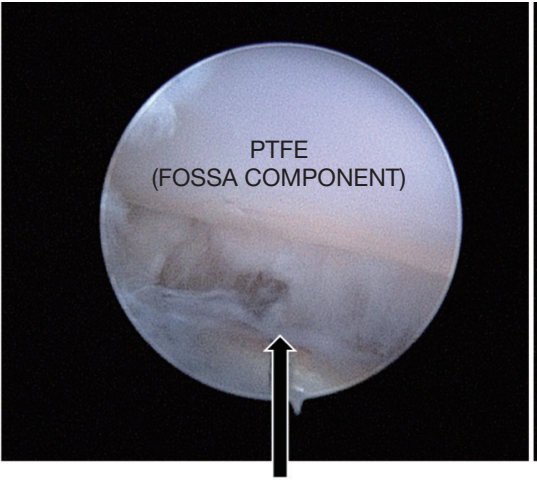

CHROME COBALT CONDYLE

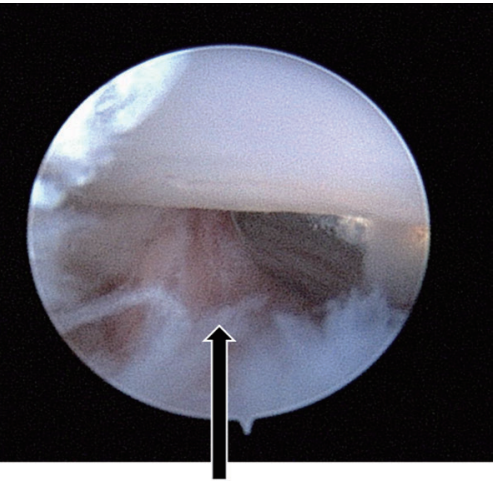

SYNOVIAL PLICA

Figure 14 Advanced operative arthroscopy on a patient with a failing total joint prosthesis. Fibrotic tissue was lysed and debrided to create a mobile joint space with polytetrafluoroethylene (PTFE) fossa and chrome cobalt condyle clearly visible. An inflamed fibrotic synovial plica was released and treated with a subsynovial steroid injection.

total joint prostheses has been described with encouraging early results in a limited population (65). The operative skills required for procedures such as these are quite challenging, as the anatomic landmarks are unlike those encountered with routine TMJ arthroscopy (Figure 14). Due to risk of damage to the prosthesis, operative maneuvers must be modified to ensure the prevention of further harm.

\section{What is the best evidence in the literature?}

Continued review of the evidence-based literature on arthroscopic temporomandibular surgery is necessary and will influence the intra-articular procedures that we will be performing in the future. Although randomized controlled clinical trials are lacking with respect to TMJ treatment in general, including surgery, there is overwhelming evidence that reported outcomes and success rates repeatedly are in the range of $80-90 \%$.

Successful outcomes are routine with arthroscopy from the most basic L\&L, to the most advanced procedures such as discopexy. One study compared outcomes of basic L\&L with various advanced operative techniques in 344 patients with chronic closed lock (66). Advanced arthroscopy included challenging techniques such as discopexy, lateral pterygoid myotomy, motorized shaving debridement and other operative maneuvers. There were no differences in outcomes between $\mathrm{L} \& \mathrm{~L}$ and advanced arthroscopy, both techniques resulting in decreased pain and increased MIO. In 1992, early in our experience with arthroscopy, a 6-year retrospective multicenter study of arthroscopy on 4,832 joints using a variety of techniques by 6 different surgeons revealed an overall success rate of approximately $90 \%$ with increased range of motion, decreased pain and increased function (67). After three decades of experience with advances in arthroscopy, success rates of $80-90 \%$ are consistently reported irrespective of arthroscopic technique.

In conclusion, the surgeon should choose the operative technique which can be performed with the least disruption to surrounding tissues, focused on diagnosis and treatment of pathologic damaged intra-articular tissues that impair TMJ function. Regardless of the technique used, basic operative arthroscopic principles include the removal of impediments to joint mobility, such as adhesions, osteoarthritic cartilage, and mobilization of the articular disc. Localized pain, swelling and impaired joint lubrication requires operative techniques to reduce inflamed synovial tissues. The importance of obtaining tissue for histopathologic examination, particularly when there are atypical intra-articular findings is necessary to establish an accurate diagnosis. Direct visualization of pathologic intra-articular tissues is a major factor in determining the operative technique, with the goal of removal and/ or treatment of these tissues to restore joint function. A major challenge for the future is to ensure that oral and maxillofacial surgeons are appropriately trained to develop arthroscopic skills so that this minimally invasive modality can continue as an essential surgical treatment that greatly benefits our patients.

\section{Predictions for the future}

Future advances in intra-articular operative arthroscopy 


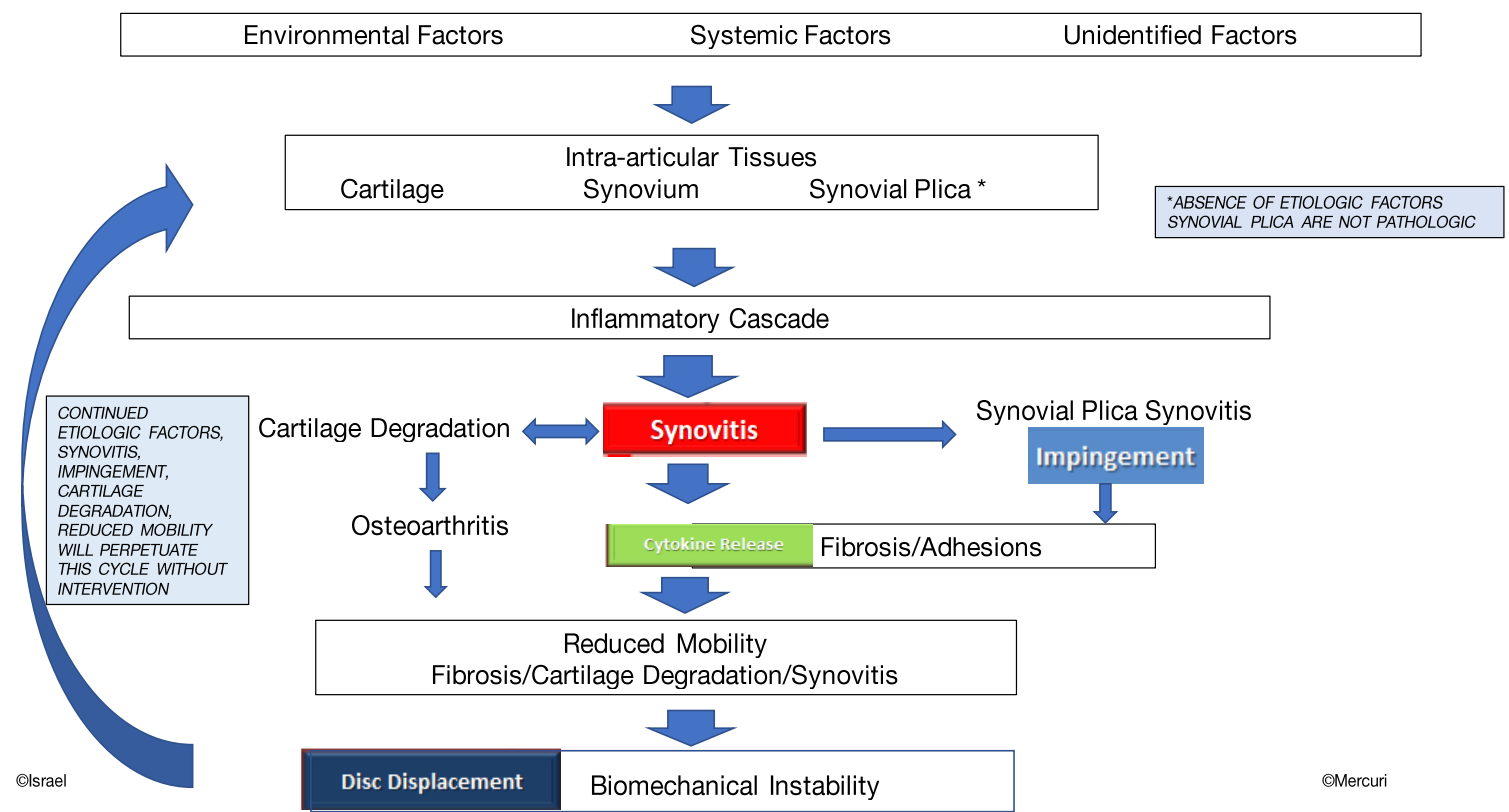

Figure 15 Pathogenic pathways leading to temporomandibular joint biomechanical instability often manifested by disc displacement, impaired mobility or hypermobility. Environmental etiologic factors include chronic joint overload or trauma. Genetic factors contribute to systemic and atypical arthropathies.

will be based on synovial fluid and tissue research on pathogenesis, which began in the 1990's $(41,43,44,51,52)$, focusing on etiologic factors, the cascade of biochemical events causing failure of joint tissue and ultimately leading to biomechanical instability. Recently a hypothesis of pathogenic pathways leading to biomechanical instability has been proposed (Figure 15), representing a summary of past and ongoing research, on the biochemical mechanisms of disease (Mercuri LG and Israel H, personal communication 2020). Arthroscopic surgery in the future will enable us to continue to obtain tissue and synovial fluid samples to define pathogenesis and develop advanced cellbased and drug-based therapies more clearly. Technological advances in arthroscopic instrumentation will lead to refinement of arthroscopic techniques improving treatment of diseased intra-articular tissues to restore function.

Virtual reality (VR) simulation education has the potential to provide enhanced arthroscopic skills training of surgeons. VR simulation training of orthopedic surgeons in hip, knee and shoulder arthroscopy has been reported and remains promising. Although VR has been viewed favorably in many studies, whether it results in significantly better performance of orthopedic residents has not been established definitively (68-75). A major challenge with VR simulation training with TMJ arthroscopy relates to the small size of the joint. One study (76) concluded that knee arthroscopy VR simulation did not objectively improve wrist arthroscopy proficiency in orthopedic residents. However, one can predict that in the future as VR simulation technology advances, small joint (including TMJ) arthroscopic proficiency of residents will be enhanced.

Studies have demonstrated that TMJ synovial fluids and tissues contain pluripotential mesenchymal stem cells that can be harvested $(77,78)$. These stem cells can differentiate into chondrocytes, osteoblasts, adipocytes, and neurons providing potential promising stem cell-based treatments. Future intra-articular surgery will likely include the harvesting of stem cells from synovium leading to innovative stem cell therapies including drug development, transplantation, and tissue engineering.

Intra-articular biopsies will become increasingly more important as investigation of synovial tissues, plicae, adhesions and degenerative cartilage will not only remove failing intra-articular tissues, but also provide tissues for studying pathogenesis, ultimately leading to advances in treatment to block the progression of pathways leading to disease.

\section{Acknowledgments}

Funding: None. 


\section{Footnote}

Provenance and Peer Review: This article was commissioned by the Guest Editors (Stephen Feinberg and Louis Mercuri) for the series "Temporomandibular Joint Disorders Diagnosis and Management - What Does the Future Hold?" published in Frontiers of Oral and Maxillofacial Medicine. The article has undergone external peer review.

Conflicts of Interest: The author has completed the ICMJE uniform disclosure form (available at https://fomm. amegroups.com/article/view/10.21037/fomm-20-34/coif). The series "Temporomandibular Joint Disorders Diagnosis and Management - What Does the Future Hold?" was commissioned by the editorial office without any funding or sponsorship. Dr. Israel reports other from Therapeutic Mobilization Devices (TMD), LLC, outside the submitted work; In addition, Dr. Israel has a patent E-Z Flex Passive Motion Jaw Exerciser issued. The author has no other conflicts of interest to declare.

Ethical Statement: The author is accountable for all aspects of the work in ensuring that questions related to the accuracy or integrity of any part of the work are appropriately investigated and resolved.

Open Access Statement: This is an Open Access article distributed in accordance with the Creative Commons Attribution-NonCommercial-NoDerivs 4.0 International License (CC BY-NC-ND 4.0), which permits the noncommercial replication and distribution of the article with the strict proviso that no changes or edits are made and the original work is properly cited (including links to both the formal publication through the relevant DOI and the license). See: https://creativecommons.org/licenses/by-nc-nd/4.0/.

\section{References}

1. Chung KC, Swanson JA, Schmitz D, et al. Introducing evidence-based medicine to plastic and reconstructive surgery. Plast Reconstr Surg 2009;123:1385-9.

2. Sato S, Sakamoto M, Kawamura H, et al. Longterm changes in clinical signs and symptoms and disc position and morphology in patients with nonreducing disc displacement in the TMJ. J Oral Maxillofac Surg 1999;57:23-9.

3. Sato S, Goto S, Kawamura H, et al. The natural course of nonreducing disc displacement of the TMJ: relationship of clinical findings at initial visit to outcome after 12 months without treatment. J Orofac Pain 1997;11:315-20.

4. Kurita K, Westesson PL, Yuasa H, et al. Natural course of untreated symptomatic TMJ disc displacement without reduction. J Dent Res 1998;77:361-5.

5. Murakami K, Kaneshita S, Kanoh C, et al. Ten-year outcome of nonsurgical treatment for the internal derangement of the TMJ with closed lock. Oral Surg Oral Med Oral Pathol Oral Radiol Endod 2002;94:572-5.

6. Minakuchi H, Kuboki T, Matsuka Y, et al. Randomized controlled evaluation of non-surgical treatments for TMJ anterior disk displacement without reduction. J Dent Res 2001;80:924-8.

7. Lundh H, Westesson L, Eriksson L, et al. TMJ disk displacement without reduction. Treatment with flat occlusal splint versus no treatment. Oral Surg Oral Med Oral Pathol 1992;73:655-8.

8. Schiffman EL, Velly AM, Look JO, et al. Effects of four treatment strategies for TMJ closed lock. Int J Oral Maxillofac Surg 2014;43:217-26.

9. Laskin D. Surgical management of internal derangements. In: Laskin DM, Greene CS, Hylander WL. editors. Temporomandibular disorders: an evidenced-based approach to diagnosis \& treatment. Quintessence Publishing, 2006:469-81.

10. Reston JT, Turkelson CM. Meta-analysis of surgical treatments for temporomandibular articular disorders. J Oral Maxillofac Surg 2003;61:3-10.

11. Al-Moraissi EA. Arthroscopy versus arthrocentesis in the management of internal derangement of the TMJ: a systematic review and meta-analysis. Int J Oral Maxillofac Surg 2015;44:104-12.

12. Kircos LT, Ortendahl DA, Mark AS, et al. Magnetic resonance imaging of the TMJ disc in asymptomatic volunteers. J Oral Maxillofac Surg 1987;45:852-54.

13. Katzberg RW, Westesson PL, Tallents RH, et al. Anatomic disorders of the TMJ disc in asymptomatic subjects. J Oral Maxillofac Surg 1996;54:147-53.

14. Takahara N, Nakagawa S, Sumikura K, et al. Association of Temporomandibular Joint Pain According to Magnetic Resonance Imaging Findings in Temporomandibular Disorder Patients. J Oral Maxillofac Surg 2017;75:1848-55.

15. Takahashi T, Nagai H, Seki H, et al. Relationship Between Joint Effusion, Joint Pain, and Protein Levels in Joint Lavage Fluid of Patients with Internal Derangement and Osteoarthritis of the TMJ. J Oral Maxillofac Surg 1999;57:1187-93.

16. Israel HA. Internal Derangement of the TMJ: New 
Perspectives on an Old Problem. Oral \& Maxillofacial Surgery Clinics of North America 2016;28:313-33.

17. Peck CC, Goulet JP, Lobbezoo F, et al. Expanding the taxonomy of the diagnostic criteria for temporomandibular disorders. J Oral Rehabil 2014;41:2-23.

18. American Society of TMJ Surgeons Guidelines for Diagnosis and Management of Disorders Involving the TMJ and Related Musculoskeletal Structures. 2001.

19. Kim YK, Im JH, Chung H, et al. Clinical application of ultrathin arthroscopy in the TMJ for the treatment of closed lock patients. J Oral Maxillofac Surg 2009;67:1039-45.

20. Hamada Y, Kondoh T, Holmlund A, et al. Cytokine and clinical predictors for treatment outcome of visually guided TMJ irrigation in patients with chronic closed lock. J Oral Maxillofac Surg 2008;66:29-34.

21. Hamada Y, Kondoh T, Holmlund A, et al. Visually guided TMJ irrigation in patients with chronic closed lock: clinical outcome and its relationship to intra-articular morphologic changes. Oral Surg Oral Med Oral Pathol Oral Radiol Endod 2003;95:552-8.

22. Vos LM, Huddleston Slater JJ, Stegenga B. Lavage therapy versus nonsurgical therapy for the treatment of arthralgia of the TMJ: a systematic review of randomized controlled trials. J Orofac Pain 2013;27:171-9.

23. Al-Belasy FA, Dolwick FM. Arthrocentesis for the treatment of TMJ closed lock: a review article. Int J Oral Maxillofac Surg 2007;36:773-82.

24. Monje-Gil F, Nitzan D, Gonzalez-Garcia R. TMJ arthrocentesis. Review of the literature. Med Oral Patol Oral Cir Bucal 2012;17:e575-81.

25. Moses JJ, Poker I. TMJ arthroscopy: an analysis of 237 patients. J Oral Maxillofac Surg 1989;47:790-4.

26. Moses JJ, Sartoris D, Glass R, et al. The effect of arthroscopic lysis and lavage of the superior joint space on the TMJ disc position and mobility. J Oral Maxillofac Surg 1989;47:674-8.

27. Miyamoto H, Sakashita H, Miyata M, et al. Arthroscopy of the TMJ: comparison of two successful techniques. Br J Oral Maxillofac Surg 1999;37:397-400.

28. Dimitroulis G. A review of 56 cases of chronic closed lock treated with TMJ arthroscopy. J Oral Maxillofac Surg 2002;60:519-24.

29. Gynther GW, Holmlund AB. Efficacy of arthroscopic lysis and lavage in patients with TMJ symptoms associated with generalized osteoarthritis or rheumatoid arthritis. J Oral Maxillofac Surg 1998;56:147-51.

30. Abboud W, Yahalom R, Givol N. Treatment of intermittent locking of the jaw in Wilkes Stage II derangement by arthroscopic lysis and lavage. J Oral Maxillofac Surg 2015;73:1466-72.

31. Smolka W, Yanai C, Smolka K, et al. Efficiency of arthroscopic lysis and lavage for internal derangement of the TMJ correlated with Wilkes classification. Oral Surg Oral Med Oral Pathol Oral Radiol Endod 2008;106:317-23.

32. Machoň V, Sedy J, Klima K, et al. Arthroscopic lysis and lavage in patients with temporomandibular anterior disc displacement without reduction. Int J Oral Maxillofac Surg 2012;41:109-13.

33. Sorel B, Piecuch JF. Long-term evaluation following TMJ arthroscopy lysis and lavage. Int J Oral Maxillofac Surg 2000;29:259-63.

34. Leibur E, Jagur O, Muursepp P, et al. Long-term evaluation of arthroscopy with lysis and lavage of TMJ disorders. J Craniomaxillofac Surg 2010;38:615-20.

35. Kaneyama K, Segami N, Sato J, et al. Outcomes of 152 temporomandibular joints following arthroscopic anterolateral capsular release by holmium: YAG laser or electrocautery. Oral Surg Oral Med Oral Pathol Oral Radiol Endod 2004;97:546-51; discussion 552.

36. Moses JJ, Poker ID. TMJ arthroscopy: the endaural approach. Int J Oral Maxillofac Surg 1989;18:347-51.

37. Morey-Mas MA, Caubet-Biayna J, Varela-Sende L, et al. Sodium Hyaluronate improves outcomes after arthroscopic lysis and lavage in patients with Wilkes stage III and IV disease. J Oral Maxillofac Surg 2010;68:1069-74.

38. Israel HA, Diamond B, Saed-Nejad F, et al. Correlation between arthroscopic diagnosis of osteoarthritis and synovitis of the human TMJ and keratan sulfate levels in the synovial fluid. J Oral Maxillofac Surg 1997;55:210-7.

39. Israel HA, Syrop SB. The important role of motion in the rehabilitation of patients with mandibular hypomobility: a review of the literature. Cranio 1997;15:74-83.

40. Israel HA, Diamond B, Saed-Nejad F, et al. Osteoarthritis and synovitis as major pathoses of the TMJ: comparison of clinical diagnosis and arthroscopic morphology. J Oral Maxillofac Surg 1998;56:1023-7; discussion 1028.

41. Stegenga B, de Bont LG, Boering G. Osteoarthrosis as the cause of craniomandibular pain and dysfunction: a unifying concept. J Oral Maxillofac Surg 1989;47(249-56.

42. Israel HA. The use of arthroscopy for treatment of TMJ disorders. J Oral Maxillofac Surg 1999;57:579-82.

43. Ratcliffe A, Israel H, Saed-Nejad F, et al. Proteoglycans in the synovial fluid of the TMJ as an indicator of changes in cartilage metabolism during primary and secondary osteoarthritis. J Oral Maxillofac Surg 1998;56:204-8.

44. Israel HA. From Arthroscopy to the Chemistry of the 
Joint. J Oral Maxillofac Surg 2007;65:13.

45. Israel HA. Technique for placement of a discal traction suture during TMJ arthroscopy. J Oral Maxillofac Surg 1989;47:311-3.

46. McCain JP, Podrasky AE, Zabiegalski NA. Arthroscopic disc positioning and suturing: a preliminary report. J Oral Maxillofac Surg 1992;50:568-79; discussion 579-80.

47. McCain JP, Hossameldin RH, Srouji S, et al. Arthroscopic discopexy is effective in managing TMJ internal derangement in patients with Wilkes stage II and III. J Oral Maxillofac Surg 2015;73:391-401.

48. Zhang SY, Liu XM, Yang C, et al. New arthroscopic disc repositioning and suturing technique for treating internal derangement of the temporomandibular joint: part II-magnetic resonance imaging evaluation. J Oral Maxillofac Surg 2010;68:1813-7.

49. Zhang S, Huang D, Liu X, et al. Arthroscopic treatment for intra-articular adhesions of the TMJ. J Oral Maxillofac Surg 2011;69:2120-7.

50. Goizueta Adame CC, Munoz-Guerra MF. The posterior double pass suture in repositioning of the TMJ disc during arthroscopy: a report of 16 cases. J Craniomaxillofac Surg 2012;40:86-91.

51. Dijkgraaf LC, DeBont GM, Boering G, et al. The structure, biochemistry and metabolism of osteoarthritic cartilage: A review of the literature. J Oral Maxillofac Surg 1995;53:1182-92.

52. Milam SB, Schmitz JP. Molecular biology of TMJ disorders: Proposed mechanisms of disease. J Oral Maxillofac Surg 1995;53:1448-54.

53. Israel HA, Langevin CJ, Singer M, et al. The relationship between TMJ synovitis and adhesions: pathogenic mechanisms and clinical implications for surgical management. J Oral Maxillofac Surg 2006;64:1066-74.

54. Chen MJ, Yang C, Zhang SY, et al. Use of coblation in arthroscopy of the TMJ. J Oral Maxillofac Surg 2010;68:2085-91.

55. Wilk BR, McCain JP. Rehabilitation of the temporomandibular joint after arthroscopic surgery. Oral Surg Oral Med Oral Pathol 1992;73:531-6.

56. Hakim MA, Christensin B, Ahn DY, et al. Correlation of arthroscopic and histologic findings in synovial membrane disease of the TMJ. J Oral Maxillofac Surg 2020;78:1297-303.

57. Cho J, Israel H: Does the age of a patient affect the outcome of TMJ arthroscopy? J Oral Maxillofac Surg 2017;75:1144-50.

58. Muñoz-Guerra MF, Rodríguez-Campo FJ, Escorial-
Hernández V, et al. TMJ disc perforation: long-term results after operative arthroscopy. J Oral Maxillofac Surg 2013;71:667-76.

59. Quinn JH. Arthroscopic management of TMJ disc perforations and associated advanced chondromalacia by discoplasty and abrasion arthroplasty: Preliminary results. J Oral Maxillofac Surg 1994;52:800-6.

60. Luder HU. Articular degeneration and remodeling in human TMJs with normal and abnormal disc position. J Orofac Pain 1993;7:391-402.

61. Willard VP, Arzi B, Athanasiou A. The Attachments of the TMJ Disc: A Biochemical and Histological Investigation. Arch Oral Biol 2012;57:599-606.

62. Nakao Y, Konno-Nagasaka M, Toriya N, et al. Proteogylcan Expression is Influenced by Mechanical Load in TMJ Discs. J Dent Res 2015;94:93-100.

63. McCain JP, Hossameldin R, Glickman AG. Preliminary clinical experience and outcome of TMJ arthroscopic chemical contracture procedure in dislocation patients. $\mathrm{J}$ Oral Maxillofac Surg 2014;72:e16-7.

64. Ybema A, De Bont LGM, Spijkervet FKL. Arthroscopic Cauterization of Retrodiscal Tissue as a Successful Minimal Invasive Therapy in Habitual TMJ. Luxation. Int J Oral Maxillofac Surg 2013;42:376-9.

65. Davis CM, Hakim M, Choi D, et al. Early Clinical Outcomes of the Arthroscopic Management of the Failing Alloplastic TMJ Prosthesis. J Oral Maxillofac Surg 2020;78:903-7.

66. González-García R, Rodriguez-Campo FG, Monje F, et al. Operative versus simple arthroscopy for chronic closed lock of the TMJ: a clinical study of 344 arthroscopic procedures. Int J Oral Maxillofac Surg 2008;37:790-6.

67. McCain JP, Sanders B, Koslin MG, et al. Temporomandibular joint arthroscopy: a 6-year multicenter retrospective study of 4,831 joints. J Oral Maxillofac Surg 1992;50:926-30.

68. Rahm S, Wieser K, Bauer DE, et al. Efficacy of standardized training on a virtual reality simulator to advance knee and shoulder arthroscopic motor skills. BMC Musculoskelet Disord 2018;19:150.

69. Wang KC, Bernardoni ED, Cotter EJ, et al. Impact of simulation training on diagnostic arthroscopy performance: a randomized controlled trial. Arthrosc Sports Med Rehabil 2019;1:e47-57.

70. Hurmusiadis V, Rhode K, Schaeffter T, et al. Virtual arthroscopy trainer for minimally invasive surgery. Stud Health Technol Inform 2011;163:236-8,

71. Bauer DE, Wieser K, Aichmair A, et al. Validation 
of a virtual reality-based hip arthroscopy simulator. Arthroscopy 2019;35:789-95.

72. Madan SS, Pai DR. Role of simulation in arthroscopy training. Simul Healthc 2014;9:127-35.

73. Bartlett JD, Lawrence JE, Khanduja V. Virtual reality hip arthroscopy simulator demonstrates sufficient face validity. Knee Surg Sports Traumatol Arthrosc 2019;27:3162-67.

74. Middleton RM, Alvand A, Roberts PG, et al. Simulationbased training platforms for arthroscopy: a randomized comparison of virtual reality learning to benchtop learning. Arthroscopy 2017;33:996-1003.

75. Bartlett JD, Lawrence JE, Stewart ME, et al. Does virtual

doi: $10.21037 /$ fomm-20-34

Cite this article as: Israel HA. Intra-articular operative temporomandibular joint arthroscopy. Front Oral Maxillofac Med 2021;3:3. reality simulation have a role in training trauma and orthopaedic surgeons? Bone Joint J 2018;100-B:559-65.

76. Ode G, Loeffler B, Chadderdon, RC, et al. Wrist arthroscopy: can we gain proficiency through knee arthroscopy simulation. J Surg Educ 2018;75:1664-72.

77. Zhang S, Yap AU, Toh WS. Stem cells for temporomandibular joint repair and regeneration. Stem Cell Rev Rep 2015;11:728-42.

78. Koyama N, Bessho K. Pluripotency of mesenchymal cells derived from synovial fluid in patients with temporomandibular joint disorder. Life Sci 2011;89:741-7. 